\title{
Cerebral Autosomal Dominant Arteriopathy with Subcortical Infarcts and Leukoencephalopathy: An Imaging Mimic of Multiple Sclerosis
}

\author{
A Report of Two Cases
}

\author{
Tarun Pandey $^{\mathrm{a}}$ Shefeek Abubacker ${ }^{\mathrm{b}}$ \\ Departments of Clinical Radiology, ${ }^{\mathrm{a}} \mathrm{Al}-$ Razi and ${ }^{\mathrm{b}} \mathrm{Al}-\mathrm{Sabah}$ Hospitals, Kuwait
}

\section{Key Words}

Cerebral autosomal dominant arteriopathy with subcortical infarcts and leukoencephalopathy (CADASIL) • Magnetic resonance imaging $\cdot$ Stroke, adult

\begin{abstract}
Objective and Importance: To describe the imaging findings of two cases of cerebral autosomal dominant arteriopathy with subcortical infarcts and leukoencephalopathy (CADASIL) mimicking multiple sclerosis. Clinical Presentation and Intervention: Two cases presenting with neurological signs and symptoms were referred for magnetic resonance imaging (MRI) evaluation of the brain. Case 1 was a 36-yearold female patient presenting with recurrent headaches and recent onset numbness in the fingers of the right hand. Neurological examination showed a mild sensory deficit in the right hand. Case 2 was a 31-year-old female patient presenting with attacks of right-sided numbness of the face and body. The neurological examination revealed a sensory loss in the face and brisk deep tendon reflexes. Routine MRI sequences showed two types of lesions in both cases: 'punctate' hyperintense lesions on $\mathrm{T}_{2}$-weighted images $\left(\mathrm{T}_{2} \mathrm{Wl}\right) / \mathrm{flu}$ id-attenuatedinversionrecovery(FLAIR)images, hypointense on $\mathrm{T}_{1}$-weighted images $\left(\mathrm{T}_{1} \mathrm{WI}\right)$ and 'diffuse' white matter lesions, hyperintense on $T_{2} \mathrm{WI} / \mathrm{FLAIR}$ sequences and isointense
\end{abstract}

to hypointense on $T_{1} W l$. All lesions showed no contrast enhancement. Both cases were previously clinically and radiologically diagnosed as multiple sclerosis. There was a strong family history consistent with recurrent infarctions in other family members of both patients. Both cases were later diagnosed as CADASIL by skin biopsy/genetic linkage studies and follow-up. Conclusion: The cases showed that CADASIL causes stroke-like episodes in adults and can mimic multiple sclerosis on imaging. Clinical evaluation and MRI findings allow a differentiation of the two entities.

Copyright $\odot 2006$ S. Karger AG, Basel

\section{Introduction}

Cerebral autosomal dominant arteriopathy with subcortical infarcts and leukoencephalopathy (CADASIL) is a relatively recently described autosomal dominant disorder caused by mutations of the notch 3 gene located on chromosome 19q12 which encodes a notch 3 receptor protein [1]. The disease was probably first described as early as 1955 [2] and 1977 [3] and has only recently been recognized as a distinct entity with more and more cases and families being reported worldwide $[4,5]$. Various mutations in the notch 3 gene cause the disease, but the mechanism remains unknown. Pathologically, the disease is

\section{KARGER}

Fax +4161306 1234

E-Mail karger@karger.ch

www.karger.com (c) 2006 S. Karger AG, Basel

1011-7571/06/0155-0391\$23.50/0

Accessible online at:

www.karger.com/mpp
Tarun Pandey

PO Box 54317

85864 Jaleeb Al-Shoyoukh (Kuwait)

Tel. +965 640 9076, Fax +965 398 2047, E-Mail drtarunpandey@hotmail.com 
characterized by a stereotypic degeneration of the arterial walls of small and medium-sized vessels with deposition in the media of a nonatheromatous, nonamyloidotic substance that under the electron microscope appears as a granular osmiophilic material pathognomonic for the disease. The nature of the granular osmiophilic material is undetermined and the pathogenesis remains to be elucidated. This deposition leads to a progressive narrowing of the blood vessels which is responsible for the clinical manifestations of the disease [6]. Clinically, CADASIL only involves the brain and is a type of hereditary stroke and dementia characterized by recurrent subcortical ischemic strokes starting in mid adulthood, leading to severe motor disability, pseudobulbar palsy and subcortical dementia. Clinical findings depend on the age at onset. The early form of the illness is found in young patients, generally less than 30 years old, and the main clinical manifestation is a migraine headache with subcortical lesions in the white matter, while in the later form ischemic events and behavioral symptoms are predominant. There is so far no specific treatment and death occurs after a mean of 20 years [4].

There is considerable clinical and imaging similarity between CADASIL and multiple sclerosis (MS). Both cause recurrent neurological deficits in young adults with symptom-free intervening periods and progressive neurological dysfunction due to repeated episodes. To complicate matters, a family history of MS is not uncommon. Both diseases also have similar imaging abnormalities; however, certain specific imaging markers allow a differentiation of these entities [5]. We report the imaging features of two cases of CADASIL, which to the best of our knowledge are the first reported cases of this rare disease from Kuwait.

\section{Case Reports}

\section{Case 1}

A 36-year-old Kuwaiti female patient complained of recurrent headache and numbness during the last year. She had been diagnosed as having MS previously, both clinically and on a prior magnetic resonance imaging (MRI) scan, and presented with a recent onset of numbness in the fingers of the right hand. The neurological examination showed a mild sensory loss to light touch in a glove distribution in the right hand. There was no clinical evidence of spinal cord involvement. The ophthalmologic evaluation was also unremarkable. A second neuroradiological evaluation was requested and a repeat MRI examination was performed using routine sequences, including fluid-attenuated inversion recovery (FLAIR) and specialized sequences like diffusion-weighted imaging (DWI) and magnetic resonance angiogra- phy. The MRI scan revealed extensive white matter abnormalities following 2 basic patterns: the first were small focal lesions that spared the cortex and were hypointense on $\mathrm{T}_{1}$-weighted images $\left(\mathrm{T}_{1} \mathrm{WI}\right)$ and hyperintense on $\mathrm{T}_{2}$-weighted images $\left(\mathrm{T}_{2} \mathrm{WI}\right) /$ FLAIR images. The second type of signal abnormality consisted of large coalescent lesions in the white matter, isointense to hypointense on $\mathrm{T}_{1} \mathrm{WI}$ and diffusely hyperintense on $\mathrm{T}_{2} \mathrm{~W}$ and FLAIR images. These lesions were most predominant in the frontal, temporal and insular regions, though they were also present in the internal and external capsules, corpus callosum, periventricular regions, centrum semiovale, basal ganglia and brainstem (fig. 1). Mild signal intensity reductions were noted within the deep cerebellar white matter, crus cerebri and thalamus. There was a relative sparing of the fronto-orbital and occipital subcortical areas. The optic nerves were normal. None of the lesions showed contrast enhancement. There was no evidence of any area of restricted diffusion on the DWI or any vascular abnormality on the magnetic resonance angiography either.

A review of other investigations showed the absence of oligoclonal bands in the cerebrospinal fluid (CSF). There was no history of hypertension and vasculitis markers were negative. The clinical picture and MRI findings were consistent with CADASIL. A review of the family history showed that the patient's mother suffered from similar episodes, was also diagnosed as having MS and had developed progressive subcortical dementia over the years. Her brother was diagnosed as having MS, too. He presented a year earlier with an episode of diplopia and a review of his MRI done at that time showed characteristic lesions of CADASIL. The diagnosis of CADASIL was subsequently confirmed by skin biopsy and genetic screening in this case as well as in her mother's and her brother's case.

Case 2

The patient was a 31-year-old Kuwaiti female. She presented with attacks of right-sided numbness of the face and body. The neurological examination revealed sensory loss in the face and brisk deep tendon reflexes more on the right side. There were no visual complaints and the ophthalmologic evaluation was within normal limits. An MRI scan done in a private radiology clinic was interpreted as MS. The CSF oligoclonal band was absent. The patient was referred to us for neuroradiological consultation and a repeat MRI scan as the diagnosis was elusive and the patient's symptoms continued. A routine contrast-enhanced MRI scan of the brain and spine together with DWI were done. White matter lesions in the brain similar in pattern and signal intensity to the ones described in case 1 with especially prominent confluent white matter lesions in both anterior temporal lobes (fig. 2) were observed. In addition, focal lesions were also seen in the cerebellum. No lesion was seen in the spinal cord or optic nerves. None of the lesions showed restricted diffusion on the DWI or any evidence of contrast enhancement. A review of the outside MRI scan of the same patient done a few months earlier showed similar abnormalities.

The family history displayed similar neurological problems in her mother and sister who were diagnosed as having MS. Thus, this patient and other members of her family showed features compatible with CADASIL which was later confirmed by genetic screening. 

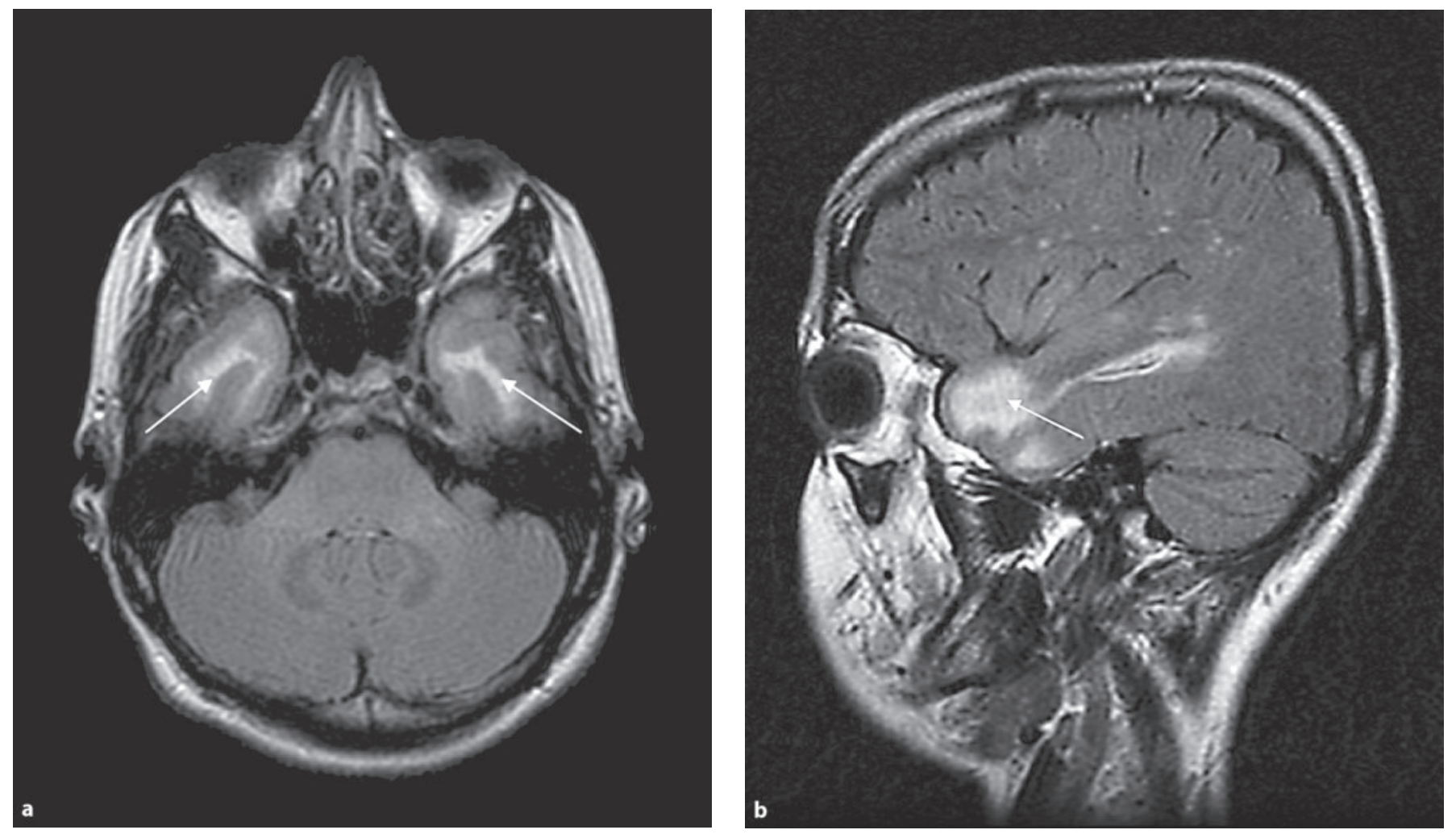

Fig. 1. MRI images of the first case. a Axial FLAIR image showing the anterior temporal lobe hyperintensities characteristic of CADASIL. b Sagittal FLAIR image of the left temporal lobe showing the temporal lobe changes more distinctly. c Sagittal FLAIR image showing the pericallosal changes mimicking MS.

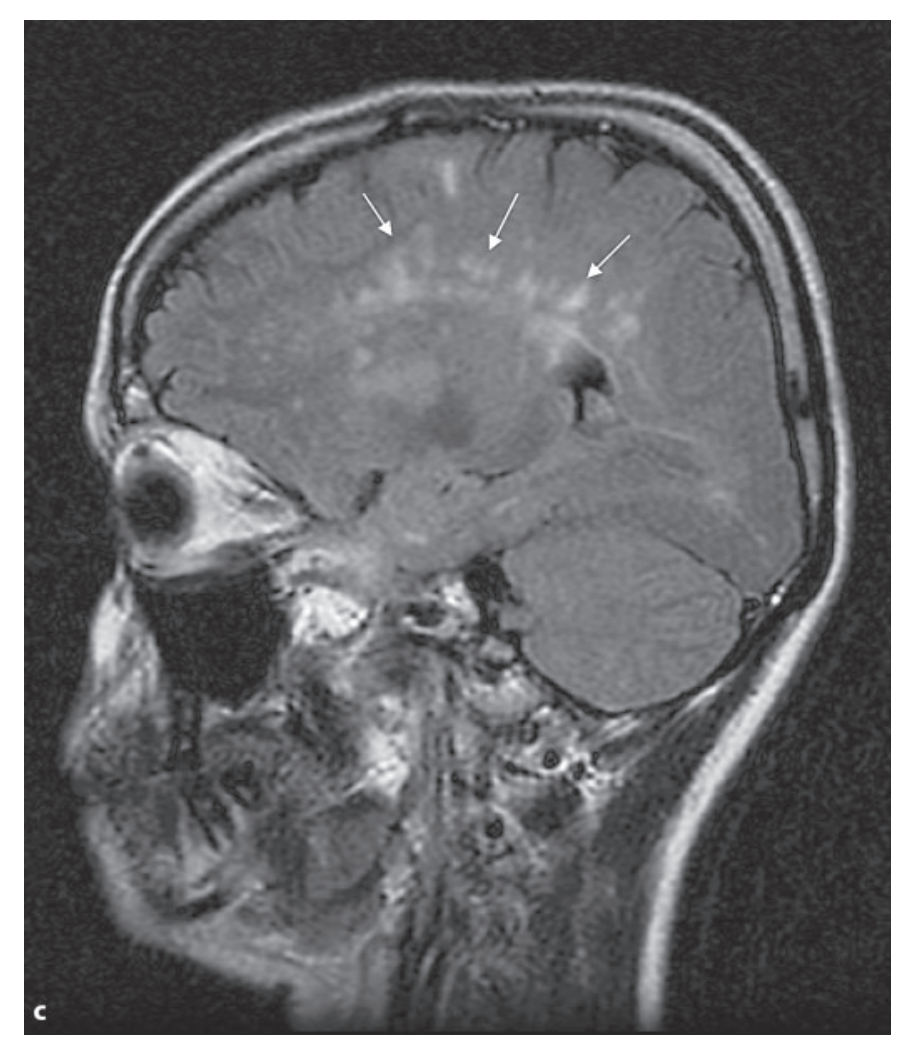



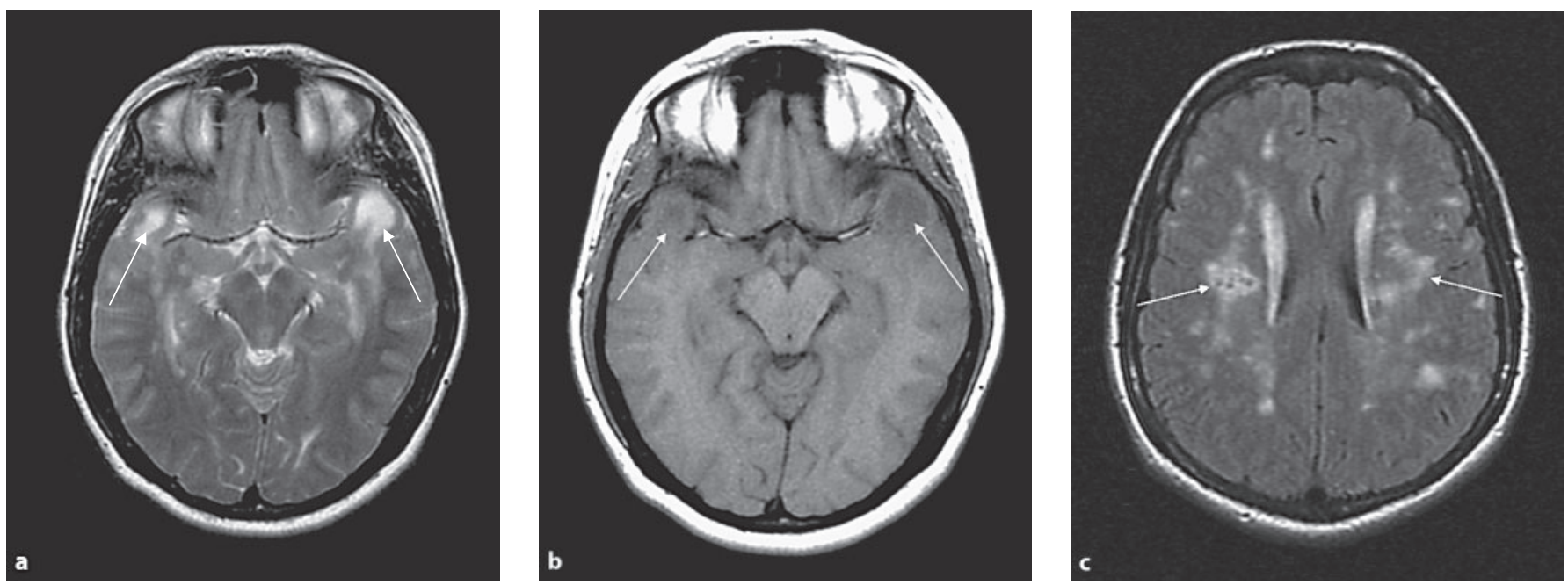

Fig. 2. MRI images of the second case. a Axial $\mathrm{T}_{2} \mathrm{WI}$ of the brain showing the characteristic hyperintense anterior temporal lobe signal. b Axial $\mathrm{T}_{1} \mathrm{WI}$ of the brain showing the hypointensity in the anterior temporal lobes. c The axial FLAIR image shows multiple focal, subcortical and deep white matter hyperintense lesions, few of which show cystic changes denoting infarction.

\section{Discussion}

CADASIL can mimic MS both clinically and on MRI (fig. 1). In fact, it was confused with MS in the initially described families with this disease [1]. The reasons for the diagnostic confusion include the presence of recurrent, remitting episodes of focal neurological deficits in both these conditions, their occurrence in relatively young individuals and intervening symptom-free periods for many years. Also, a family history of MS is not uncommon and up to 1 in 20 patients with this disease have an affected first-degree relative. On MRI, both conditions can show lesions similar in morphology and location. The typical MS lesion, Dawson's finger, which consists of cigarshaped lesions perpendicular to the corpus callosum, can also be seen in CADASIL along with similar lesions in the callososeptal interface, peritrigonal white matter, brainstem and centrum semiovale as seen in the present cases (fig. 1c, 2c). Specialized sequences like the DWI can show a bright signal due to restricted diffusion in the acute phase of both these conditions and therefore is not so helpful in the differentiation of these entities. Though CADASIL is a vascular disease, the digital subtraction angiogram is characteristically normal. Not surprisingly, the magnetic resonance angiography performed in the first case was normal. Contrast enhancement of the MS lesion(s), if present, can differentiate it from CADASIL; however, an absence of enhancing lesion(s) does not help.
For CADASIL, however, there are many potential clinical and imaging distinguishing features including an autosomal dominant mode of inheritance, a positive family history of stroke, vascular dementia and migraine headache, and an absence of optic neuritis and spinal cord manifestations [7]. Recent reports and studies have also shown a few specific MRI findings in this disease which have been described as radiological markers of CADASIL. These include hyperintensities in the white matter of the anterior temporal lobes and also frequent involvement of the external capsules [8] as seen in our two cases. Interestingly, these findings also distinguish CADASIL from the other common imaging mimic, sporadic subcortical arteriosclerotic encephalopathy, which can also present with multiple infarcts [9]. Temporal pole involvement was present in $95 \%$ of all patients with CADASIL, but in no patient with ischemic leukoaraiosis [8].

There may be predilection for temporal lobe white matter because this region is differently vulnerable to ischemia in CADASIL compared to other areas of the brain [10]. Also the patients of sporadic subcortical arteriosclerotic encephalopathy tend to be older and have a history of hypertension unlike CADASIL. The penetrance of CADASIL appears complete between 30 and 40 years of age based on MRI data which indeed is a very specific diagnostic tool [11].

The other conditions in the differential diagnosis of CADASIL include mitochondrial encephalomyelopathy 
with lactic acidosis and stroke-like episodes (MELAS), primary angiitis of the central nervous system and hypercoagulable states like antiphospholipid syndrome and protein $S$ deficiency. Although these conditions can cause recurrent strokes or stroke-like episodes in young people, they can be differentiated from CADASIL both clinically and on imaging. Cortical involvement is not seen in CADASIL but is a feature of all these conditions. Of note is the fact that the lesions in MELAS, unlike CADASIL, tend to disappear with clinical improvement. The presence of angiographic abnormalities is seen in primary angiitis and hypercoagulable states, a feature not seen with CADASIL. Dural sinus thrombosis may also be seen with hypercoagulable states on imaging.

The pathological hallmark of CADASIL is the presence of granular, electron-dense, osmiophilic material adjacent to the basement membrane of smooth muscle cells of arterioles on electron microscopy. These ultrastructural changes are seen in both skin and brain vessels, indicating that CADASIL is a systemic vasculopathy, though the clinical features are usually confined to the central nervous system [6].

The cases we reported were erroneously misinterpreted as MS initially. This is not only because of the similar clinical and imaging features of the diseases and the high prevalence of MS in our population, but also because of a lack of awareness of this relatively recent entity described as CADASIL. An autosomal dominant pattern of inheritance in CADASIL can be discovered by obtaining a thor- ough family history but one must be cautious as MS patients can also have a positive family history and can have superficial resemblance to CADASIL. In fact, the seemingly apparent family history of MS was a confounding factor in the first case of our report. Similarly, a thorough history and examination can detect the presence of optic neuritis and/or the spinal cord manifestations of MS. But in individual cases, the absence of such signs and symptoms does not allow differentiation between the two conditions. Moreover, spinal cord disease has been reported in CADASIL [12]. Also, the presence of oligoclonal bands in the CSF, though exceedingly rare, can be seen in CADASIL [11]. More often than not, the definite diagnosis of CADASIL can be made on imaging if care is taken to avoid the misleading results while looking for the characteristic findings. This strategy was applied in our cases where on review of the MRI scans the characteristic radiological markers of anterior temporal lobe hyperintensities and external capsule abnormalities were detected and a correct diagnosis of CADASIL was made.

\section{Conclusion}

The cases showed that CADASIL causes stroke-like episodes in adults and can mimic MS on imaging. Clinical evaluation and MRI findings allow differentiation of the two entities.

\section{References}

$>1$ Tournier-Lasserve E, Iba-Zizen MT, Romero N, Bousser MG: Autosomal dominant syndrome with stroke-like episodes and leukoencephalopathy. Stroke 1991;22:1297-1302.

2 Van Bogaert L: Encéphalopathie sous-corticale progressive (Binswanger) à évolution rapide chez deux sœurs. Med Hellen 1955; 24:961-972.

$>3$ Sourander P, Walinder J: Hereditary multiinfarct dementia: morphological and clinical studies of a new disease. Acta Neuropathol (Berl) 1977;39:247-254.

$\checkmark 4$ Chawda SJ, De Lange RP, Hourihan MD, Halpin SF, St Clair D: Diagnosing CADASIL using MRI: evidence from families with known mutations of Notch 3 gene. Neuroradiology 2000;42:249-255.

$\checkmark 5$ Kimper-Karl AC, Jensen HB, Kristensen O: CADASIL and multiple sclerosis. Ugeskr Laeger 2006;168:702-703.
6 Ebke M, Dichgans M, Bergmann M, Voelter HU, Rieger P, Gasser T, Schwendemann G: CADASIL: skin biopsy allows diagnosis in early stages. Acta Neurol Scand 1997;95:351357.

7 Vahedi K, Tournier-Lasserve E, Chabriat H, Bousser MG: An additional monogenic disorder that masquerades as multiple sclerosis. Am J Med Genet 1996;65:357-358.

-8 O'Sullivan M, Jarosz JM, Martin RJ, Deasy N, Powell JF, Markus HS: MRI hyperintensities of the temporal lobe and external capsule in patients with CADASIL. Neurology 2001; 56:628-634.

$>9$ Auer DP, Putz B, Gossl C, Elbel G, Gasser T, Dichgans M: Differential lesion patterns in CADASIL and sporadic subcortical arteriosclerotic encephalopathy: MR imaging study with statistical parametric group comparison. Radiology 2001;218:443-451.
10 Chabriat H, Levy C, Taillia H, Iba-Zizen MT, Vahedi K, Joutel A, Tournier-Lasserve E, Bousser MG: Patterns of MRI lesions in CADASIL. Neurology 1998;51:452-457.

11 Chabriat H, Vahedi K, Iba-Zizen MT, Joutel A, Nibbio A, Nagy TG, Krebs MO, Julien J, Dubois B, Ducrocq X: Clinical spectrum of CADASIL: a study of 7 families. Cerebral autosomal dominant arteriopathy with subcortical infarcts and leukoencephalopathy. Lancet 1995;346:934-939.

12 Hutchinson M, O’Riordan J, Javed M, Quin E, Macerlaine D, Wilcox T, Parfrey N, Nagy TG, Tournier-Lasserve E: Familial hemiplegic migraine and autosomal dominant arteriopathy with leukoencephalopathy (CADASIL). Ann Neurol 1995;38:817-824. 